\title{
Effect of Risperidone and Fluoxetine on the Movement and Neurochemical Changes of Zebrafish
}

\author{
Maria Jimena Prieto ${ }^{1,2^{*}}$, Hector Carreño Gutierrez ${ }^{3}$, Rosario Arévalo Arévalo ${ }^{3}$, \\ Nadia S. Chiaramoni ${ }^{1,2}$, Silvia del Valle Alonso ${ }^{1,2}$ \\ ${ }^{1}$ Biomembrane Laboratory, Department of Science and Technology, National University of Quilmes, Buenos Aires, Argentina \\ ${ }^{2}$ IMBICE-CONICET, Buenos Aires, Argentina \\ ${ }^{3}$ Department of Cell Biology and Pathology, Institute of Neuroscience of Castilla y Leon, School of Medicine, \\ University of Salamanca, Salamanca, Spain \\ Email: *jprieto@unq.edu.ar
}

Received September 20, 2012; revised October 23, 2012; accepted November 4, 2012

\begin{abstract}
Brain developmental disorders in humans, including Autism Spectrum Disorders (ASD) and Down's syndrome, have been linked to increased serotonin levels. This work was designed to study changes in serotonin levels in the early stages of development with two classes of antipsychotic drugs: Risperidone, a drug that blocks serotonin and dopamine receptors, and fluoxetine, a serotonin reuptake inhibitor. The use of antipsychotic drugs is a solid choice to study the decrease and increase of these neurotransmitters and their influence on development. The study of these parameters will give an idea of the effects of serotonin in early developmental stages. To this end, we examined the effects of risperidone and fluoxetine on the locomotor activity, heart rate and brain development of zebrafish larvae. Our results showed that in larvae exposed to fluoxetine alone, swimming was significantly increased at $9 \mathrm{dpf}$ (days post-fertilization). Erratic and abnormal movements were observed suggesting a toxic effect of fluoxetine. No erratic swimming was observed in larvae treated with fluoxetine plus risperidone. Both drugs presented morphological changes in dopaminergic neurons and mononeurons. Exposure to fluoxetine plus risperidone indicated possible reversal effects. Studies in zebrafish allow obtaining new insights into the side effects of these drugs as well as into the brain control of locomotor activity. Testing several drug-induced changes in behavior and serotonin levels is one of the experimental approaches for screening a new therapeutically relevant compound, and thus, merits further research.
\end{abstract}

Keywords: Zebrafish; Risperidone; Fluoxetine; Serotonin; Behavioral

\section{Introduction}

Serotonin has shown to play a role in regulating brain and spinal cord development before assuming its role as a neurotransmitter in the mature central nervous system (CNS) $[1,2]$. Serotonin plays an important role in modulating motor pathways in all vertebrates. The early appearance of serotonin neurons and their wide distribution of extra-synaptic and synaptic terminals are consistent with the notion that serotonin acts as a growth factor or a signal molecule for multiple developmental mechanisms.

Increased serotonin levels have also been linked to brain developmental disorders in humans, including Autism Spectrum Disorders (ASD) and Down's syndrome [3-7]. ASD include different neurodevelopmental disorders that become manifest mainly in the earlier years of life [8] and affect the development of language, communication, and reciprocal social interaction [9], with an incidence of 1 out of 150 individuals [10].

*Corresponding author.
The main problem with the theory of serotonin as a primary cause of autism is that treatments which further increase serotonin levels seem to improve some symptoms of autism, such as obsessiveness and social relatedness [11], while the depletion of tryptophan, a serotonin precursor, seems to exacerbate autistic symptoms such as flapping, banging and self-hitting, rocking and increased anxiety [12].

On the other hand, a recent clinical study has reported that children exposed to therapeutic levels of a selective serotonin reuptake inhibitor (SSRI), such as is the case of fluoxetine, in the third trimester of pregnancy, scored lower in psychomotor and behavioral tests administered at 6 - 40 months of age compared to children who were not exposed to a SSRI during pregnancy [13]. Delays on psychomotor development and changes in movement and control at these later ages imply a more permanent problem in motor circuitry. This is why more studies are needed to understand the neurochemistry of serotonin in the early stages of development. 
Fluoxetine has many characteristics that make it a useful tool to study increases in serotonin neurotransmission. Fluoxetine is one of several SSRIs used for treatment of clinical depression, anxiety disorder, obsesssivecompulsive disorder, and bulimia. It is also one of the most heavily prescribed antidepressant drugs worldwide. It is a potent and highly selective inhibitor of the transporter enzyme for serotonin reuptake at the presynaptic membrane, causing an increase in serotonin concentrations at postsynaptic receptor sites [14].

On the other hand, atypical antipsychotic drugs, such as risperidone, 3-[2-[4-(6-fluoro-1,2-benzisoxazol-3-yl)-1piperidinyl]ethyl]-6,7,8,9-tetrahydro-2-methyl-4H-pyrido [1,2-a] pyrimidin-4-one (Risp), have been increasingly used to treat anxiety-related disorders in addition to their use in the treatment of psychosis and ASD. Risperidone has been reported to act therapeutically by blocking serotonin and dopamine receptors [15]. This drug is useful to study increases in serotonin neurotransmission. $\mathrm{Su}$ et al. [16] showed that risperidone inhibits N-methyl-Daspartate antagonist-induced glutamate release in the medial prefrontal cortex by blocking serotonin 5-HT2A receptors on glutamatergic terminals, leading to attenuation of the activity of cortico-subcortical glutamatergic neurons. This attenuation, in turn, decreases MK-801-induced hyper-locomotion [16].

In this work, we used the zebrafish (Danio rerio) as an ideal model for studying developmental neurobiology and other fields of biomedicine. The zebrafish is a teleost of the Cyprinid family, and has very advantageous features to be used in the laboratory: its small size (no more than $5 \mathrm{~cm}$ in adults) allows the easy maintenance of several individuals with relatively low costs; females lay a large number of eggs; embryos develop rapidly and are semitransparent 24 hours post-fertilization (hpf); and embryos have a sequenced genome and numerous mutant and transgenic lines.

It is known that serotonin plays an important role in modulating the motor output in vertebrates $[17,18]$. Serotonin neurons particularly innervate motor neurons projecting to axial muscles, which are associated with rhythmic locomotor movements. In developing zebrafish, serotonin neurons and projections appear in the hindbrain and spinal cord at about 2 days post-fertilization (dpf) [19].

Based on the above, the use of antipsychotic drugs is a solid choice to study the increase and decrease of this neurotransmitter and its influence on development. This study was designed to study changes in serotonin levels in the early stages of development induced by two classes of antipsychotic drugs: Risperidone, a drug that blocks serotonin and dopamine receptors; and fluoxetine, a serotonin reuptake inhibitor. To this end, we examined the effects of risperidone and fluoxetine on the locomotor activity, heart rate, and brain development of zebrafish larvae. Study of these parameters will give an idea of the effects caused by serotonin in early developmental stages.

\section{Materials and Methods}

\subsection{Animals}

Adult zebrafish (Danio rerio) belonging to the AB line, provided by the Department of Cell Biology and Pathology, University of Salamanca, Spain, were used as breeding individuals. The animals were kept in tanks at a temperature of $28^{\circ} \mathrm{C}$, with $14 \mathrm{~h}$ of light and $10 \mathrm{~h}$ of darkness, as previously established [20]. In this study, "embryos" refer to zebrafish prior to hatching (0 - $3 \mathrm{dpf})$, while "larvae" refer to post-hatch animals (over $3 \mathrm{dpf}$ ). Embryos were obtained from natural mating, and all embryos and larvae used in these experiments were reared at $28.5^{\circ} \mathrm{C}$ on a $14 / 10 \mathrm{~h}$ light/dark cycle in conditioned E3 medium $\left(\mathrm{NaCl} 0.29 \mathrm{~g} / \mathrm{l}, \mathrm{KCl} 0.012 \mathrm{~g}, \mathrm{CaCl}_{2} 0.036 \mathrm{~g} / \mathrm{l}\right.$ and $\mathrm{MgSO}_{4} 0.039 \mathrm{~g} / 1$ in deionized water), and $50 \mathrm{ppb} \mathrm{me}-$ thylene blue (Panreac) to inhibit fungal growth. The animals were handled following the European Union directives (86/609/EEC and 2003/65/EC) and Spanish legislation (RD 1201/2005, BOE 252/34367-91, 2005).

\section{Embryo Collection}

The evening before spawning, breeding pairs of specimens were transferred to rearing tanks. These tanks were kept at $28.5^{\circ} \mathrm{C}$. The first light stimulus after the dark cycle induced egg laying. The eggs obtained were prepared in petri dishes in E3 medium. Only the eggs that were fertilized and in good condition were selected for further treatment; the others were discarded. Egg observation was followed by stereomicroscopy (Leica Zoom, 2000).

\subsection{Exposure to Risperidone and Fluoxetine}

Risperdal tablets (Jannsen Cilag Laboratory, $2 \mathrm{mg}$ risperidone) were dissolved in E3 medium and prepared as a $5 \mu \mathrm{M}$ solution. Prozac tablets (Lilly Laboratory, $20 \mathrm{mg}$ fluoxetine) were dissolved in E3 medium and prepared as a $5 \mu \mathrm{M}$ solution. In Airhart et al. 2007 [7], zebrafish embryos were exposed to seven different fluoxetine concentrations beginning at $10 \mathrm{pf}$ and up to $11 \mathrm{dpf}$, to determine the lowest observable effective concentration (LOEC). In larvae exposed to $4.6 \mu \mathrm{M}$ (LOEC) fluoxetine for $24 \mathrm{~h}$ intervals between 4 and $5 \mathrm{dpf}$, spontaneous swimming activity was significantly depressed compared to controls and remained depressed through $14 \mathrm{dpf}$. In addition, the core neural migration raphe to the spinal cord was observed between 3 and $6 \mathrm{dpf}$ [7] and serotonin levels could affect normal development of CNS. In this respect, in this work, we selected the following exposure concentrations: 1) Risp $5 \mu \mathrm{M}$ at $4 \mathrm{dpf}$ for $24 \mathrm{~h}$; 2) Risp $5 \mu \mathrm{M}$ at $6 \mathrm{dpf}$ for $24 \mathrm{~h}$; 3 ) Prozac $5 \mu \mathrm{M}$ at $4 \mathrm{dpf}$ for $24 \mathrm{~h}$; 4) Prozac $5 \mu \mathrm{M}$ at $4 \mathrm{dpf}$ for $24 \mathrm{~h}$ and Risp $5 \mu \mathrm{M}$ at $6 \mathrm{dpf}$ for 24 
h. Controls were untreated larvae. Larvae were exposed to $5 \mu \mathrm{M}$ risperidone or fluoxetine for periods of $24 \mathrm{~h}$ at 4 or $6 \mathrm{dpf}$ and subsequently rescued into a preconditioned E3 medium (Figure 1).

\subsection{Behavioral Testing: Spontaneous Swimming Activity (SSA) Measurement}

At $4 \mathrm{dpf}$, larvae were exposed for $24 \mathrm{~h}$ in a risperidone or fluoxetine solution of $5 \mu \mathrm{M}$ and at $6 \mathrm{dpf}$, larvae were monitored daily. Animals were placed individually in a 6-well multiplate at $28^{\circ} \mathrm{C}$. After a 1 -min acclimation period, the SSA was measured as the number of times the animal crossed the gridlines in 1' under a magnifying glass (Figure 2(a)). For treatments at $4 \mathrm{dpf}$ and $6 \mathrm{dpf}$ or only at $6 \mathrm{dpf}$, at $6 \mathrm{dpf}$ larvae were monitored daily.

The data was analyzed with Prism GradPad. Experiments were performed with groups of 6 larvae and the data was collected three times per group.

\subsection{Heart Rate Measurements}

The heart rate was assessed at 8 and $10 \mathrm{dpf}$. Control and experimental zebrafish larvae were individually transferred to a depression slide with methylcellulose and placed under a binocular microscope. The heart rate was determined by counting the number of beats per $15 \mathrm{~s}$ and recorded as beats per minute (bpm). Experiments were performed thrice on three larvae per group for each time point.

\subsection{Preparation of Histological Sections}

For the fixation of samples, both treated and control animals were anesthetized with a tricaine methanesulfonate solution (MS-222, Sigma) at $0.3 \mathrm{~g} / \mathrm{l}$. They were then fixed by immersion in $4 \% \mathrm{v} / \mathrm{v}$ paraformaldehyde (PFA) in phosphate buffer $0.1 \mathrm{M}(\mathrm{PB}), \mathrm{pH} 7.4$ for $24 \mathrm{~h}$ at $4^{\circ} \mathrm{C}$. Following fixation, the PFA was removed with five 5-minute washes in PBS.

Then the samples were embedded in a mixture $1.5 \%$ of agar and $10 \%$ of sucrose (Panreac) in PB. The mixture

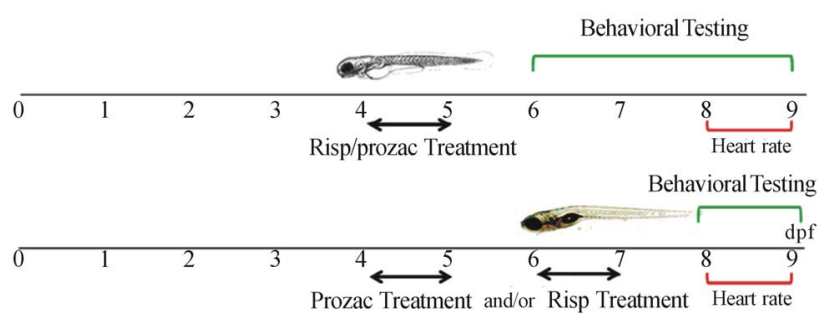

Figure 1. Timeline representing the stage specificity of the effects of fluoxetine and risperidone in the developing zebrafish. Larvae were exposed to $5 \mu \mathrm{M}$ risperidone and/or fluoxetine for $24 \mathrm{~h}$ periods from $4 \mathrm{dpf}$ and subsequently rescued into a conditioned $\mathrm{E} 3$ medium. Larvae were monitored through 9 dpf. animals were targeted. After the mixture was solidified, the larvae were cryoprotected in a $30 \% \mathrm{w} / \mathrm{v}$ sucrose solution in PB for $24 \mathrm{~h}$. Agar blocks containing cryoprotected larvae were frozen in a cryostat (Microm HM 560) and then cut at $-28^{\circ} \mathrm{C}$ in $10 \mu \mathrm{m}$ thick parasaggital serial sections, which were collected on gelatinized slides and stored at $-20^{\circ} \mathrm{C}$ until further use.

\subsection{Hematoxylin-Eosin Staining}

Histological sections were obtained as mentioned above and stained with hematoxylin-eosin to observe possible morphological changes. Briefly, the technique involved immersing the sections in eosin for $1 \mathrm{~min}$, then washing them with water for $30 \mathrm{~min}$ and incubating them for 1 min in hematoxylin. Finally, the samples were dehydrated in alcohols of increasing concentration for $5 \mathrm{~min}$ each, ending with three tanks of xylene, for 3 min each. The slides were mounted in Entellan (Merck) for analysis and storage.

Images of hematoxylin-eosin staining were taken in a light microscope (Olympus Provis AX70) coupled to a digital camera (DP70, Olympus).

Finally, to adjust the brightness and contrast of those observed directly under the microscope, Adobe ${ }^{\circledR}$ Photoshop CS2 ${ }^{\circledR}$ version 9.0 (Adobe Systems) was used.

\subsection{Immunohistochemistry in Tissue Sections}

The sections were washed three times in PB for $10 \mathrm{~min}$ to rehydrate and remove the agar and then incubated for $1 \mathrm{~h}$ at room temperature (RT) in non-immune serum (Sigma) 5.0\%, detergent Triton X-100 (Sigma) 0.2\% and $1.0 \%$ DMSO in PB.

Then, the primary antibodies were added and incubated for $24 \mathrm{~h}$ at RT. After this incubation, the exceeding antibodies were removed with three washes of $\mathrm{PB}$ and then the sections were incubated with the corresponding secondary antibodies conjugated with the appropriate fluorochrome for $1 \mathrm{~h}$ at RT. The secondary antibody was removed with three 10-min washes in $\mathrm{PB}$ with $0.4 \%$ fish gelatin (Sigma-Aldrich).

In order to mark cell nuclei, tissue sections were incubated in 4',6-diamidino-2-phenylindole (DAPI, Sigma) at a 1:10,000 concentration for $7 \mathrm{~min}$ at RT, and then washed in PB three times of 10 min each.

\section{Antibodies Used}

The antibodies used were:

The polyclonal anti-calretinin (CalR) antibody 7696 (\# 6B3 Swant, Bellinzona, Switzerland), at a 1:10,000 concentration. This antibody has been widely used in the study of the neuroanatomy of teleosts, both in adult animals [21-23] and in embryos, larvae and juveniles [23]. 
The secondary antibody was labeled with $\mathrm{Cy} 3$ (red).

The anti-tyrosine hydroxylase (TH) antibody (Incstar, Stillwater, MN, USA) [24] at a 1:1000 concentration. The secondary antibody was labeled with Cy2 (green).

The sections were examined under a microscope (Olympus Provis AX70) coupled to a digital camera (XM10, Olympus). The images were coded green (Cy2) and red (Cy3), giving yellow co-localization in merged images. The images were adjusted for brightness, contrast and colors using Adobe Photoshop 7.0 (Adobe Systems).

\subsection{Statistical Analysis}

Data was presented as a mean \pm standard deviation (S.D.) and analyzed by one-way analysis of variance (ANOVA) and Tukey's Multiple Comparison Test using GraphPad Prism v. 5. Only values with $P<0.05$ were accepted as significant.

\section{Results}

\subsection{Heart Rate Measurements}

The effect of fluoxetine and risperidone exposure on circulation was qualitatively evaluated by observing the heart rate and blood flow through the ventral aortaposterior cardinal vein channel in control versus treated larvae.

The aim of this study was to determine whether $5 \mu \mathrm{M}$ fluoxetine and/or risperidone affected blood circulation. To this end, at 4 and $6 \mathrm{dpf}$, larvae were exposed to $5 \mu \mathrm{M}$ fluoxetine and/or risperidone for $24 \mathrm{~h}$ and their heart rate monitored at 8 and 9 dpf. This data demonstrated that larvae treated with $5 \mu \mathrm{M}$ fluoxetine and/or risperidone exhibited a normal heart rate at 8 and $9 \mathrm{dpf}$ compared to controls (Table 1, $\mathrm{P}>0.05$ ).

Therefore, the effect of $5 \mu \mathrm{M}$ fluoxetine or risperidone on locomotor development in larvae was most likely based on neurological grounds rather than on cardiovascular ones.

\subsection{Behavioral Testing}

In this specific experiment, larvae were exposed to $5 \mu \mathrm{M}$ fluoxetine or risperidone at 4 and $6 \mathrm{dpf}$ for $24 \mathrm{~h}$. After fluoxetine or risperidone exposure, larvae were rescued by transfer into E4 medium. SSA in these larvae was monitored daily from 6 to 9 dpf. All SSA measurements were performed three times per larva; each data point represented the mean \pm S.D. of six larvae.

Risperidone treatment at $4 \mathrm{dpf}$ only transiently decreased SSA, with full recovery after $7 \mathrm{dpf}$.

SSA was significantly below that of controls (Figure 2(b)) in the first days after treatments $(6 \mathrm{dpf}, \mathrm{P}<0.05)$. After $7 \mathrm{dpf}$, SSA had recovered to control levels, these levels were maintained throughout the remaining experimental period (Figure 2(b)).

The same occurred in zebrafish treated with $5 \mu \mathrm{M}$ risperidone at $6 \mathrm{dpf}$ and monitored at $8 \mathrm{dpf}(8 \mathrm{dpf}, \mathrm{P}<$ $0.05)$.

A 24-h exposure to fluoxetine at $4 \mathrm{dpf}$ resulted in a significant decrease in SSA as compared to controls (6 dpf, $\mathrm{P}<$ $0.01)$. SSA recovered fully after $7 \mathrm{dpf}$, and increased significantly at $9 \mathrm{dpf}(9 \mathrm{dpf}, \mathrm{P}<0.01)$ (Figure 2 (b)); however, the movements were erratic and abnormal. It is worth mentioning that when fish were exposed to fluoxetine at $4 \mathrm{dpf}$ and then to risperidone at $6 \mathrm{dpf}$, there was no significant change in SSA with respect to control (Figure 2(b)).

\subsection{Tissue Sections}

\subsubsection{Morphological Changes}

Both treated and control animals were fixed at $10 \mathrm{dpf}$, cut in serial sections and stained, as mentioned above. A 24-h exposure to fluoxetine at $4 \mathrm{dpf}$ resulted in an observable decrease in the number of nucleated areas of the dorsalis telencephali area. The same occurred in the dorsal area of the optic tectum (Figure 3(b)) compared with the control (Figure 3(a)).

There was also a larger area in the postoptic commissure and the raphe population zone and a cellular disorganization in the latter. On the other hand, the fissure that separates the midbrain at forebrain increased in size (Figure 3(b)). All these changes were reflected as an increase in abnormal and erratic swimming in the behaveioral test. Undoubtedly, the administration of this drug to animals at $4 \mathrm{dpf}$ caused dramatic changes that persisted overtime.

In the treatment with risperidone at 4 and $6 \mathrm{dpf}$ (Figures 3(c) and (d)), an increase in the nucleated zone of

Table 1. Post-treatment heart rates in zebrafish larvae after a $24 \mathrm{~h}$ exposure to $5 \mu \mathrm{M}$ Fluoxetine or risperidone. A mean number of heart beats per minute (bpm) $( \pm$ S.D.) in control and $24 \mathrm{~h}$ fluoxetine or risperidone treated zebrafish larvae. There was no significant difference between control and fluoxetine or risperidone exposed larvae at either 8 or $9 \mathrm{dpf}(\mathrm{P}>0.05, \mathrm{n}=6)$.

\begin{tabular}{cccccc}
\hline & \multicolumn{5}{c}{ Treatment } \\
\hline Heart rate measurements & Prozac 4 dpf & Risp 6 dpf & Risp 4 dpf & Prozac 4 dpf + Risp 6 dpf & Control \\
\hline $\mathbf{8 ~ d p f}$ & $121 \pm 11$ & $128 \pm 7$ & $127 \pm 7$ & $131 \pm 6$ & $131 \pm 9$ \\
$\mathbf{9}$ dpf & $115 \pm 9$ & $125 \pm 7$ & $130 \pm 3$ & $121 \pm 10$ & $122 \pm 6$ \\
\hline
\end{tabular}




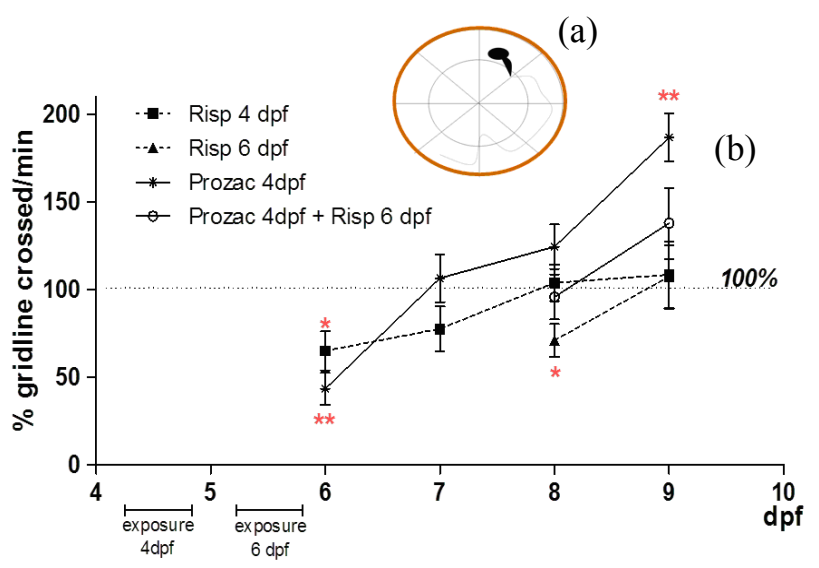

Figure 2. (a) Schematic representation of a 6-well multiplate with a dividing line for monitoring the spontaneous swimming activity (SSA); (b) Spontaneous swimming activity (SSA) measurements. All SSA measurements began at 6 dpf and were performed three times; each data point represents the mean \pm S.D. of six larvae.

the postoptic commissure and a cellular disorganization in the raphe populations zone were observed. However, these changes would not be sufficient to affect the SSA.

Zebrafish exposed to fluoxetine at $4 \mathrm{dpf}$ and then to risperidone at $6 \mathrm{dpf}$ showed no significant changes with respect to control (Figure 3(e)).

\subsubsection{Immunohistochemistry}

For the histological analysis, crop images were obtained to include the reference space area and optical angle for brain tissue. In this work, we studied two proteins to determine dopaminergic neurons, labeled with $\mathrm{TH}$, and motoneurons, labeled with CalR.

When the larvae were exposed to free risperidone, an increase in the levels of $\mathrm{TH}+$ dopaminergic neurons was observed in the dorsal region of the optic tectum of the brain (Figures 4(c) and (d)) compared with the control (Figure 4(a)). On the other hand, increases in the levels of $\mathrm{TH}+$ cells in the midbrain region were also observed. However, no significant changes in brain tissue (Figures 4(b) and (e)) were observed with fluoxetine either alone or combined with risperidone.

In the spinal cord, we found a decrease in the levels of CalR-positive motoneurons in all treatments with risperidone alone (Figures 5(c) and (d)) compared with the control (Figure 5(a)). In the case of fluoxetine, an increased width in the nerve fiber formed by the axons of motoneurons (Figure 5(b)) was observed. In the case of fish treated with the combination of fluoxetine and risperidone, there was an increased width in the nerve fiber formed by the axons of motoneurons compared with those treated with risperidone (Figure 5(e)) but less than that found in those under fluoxetine alone. (a)
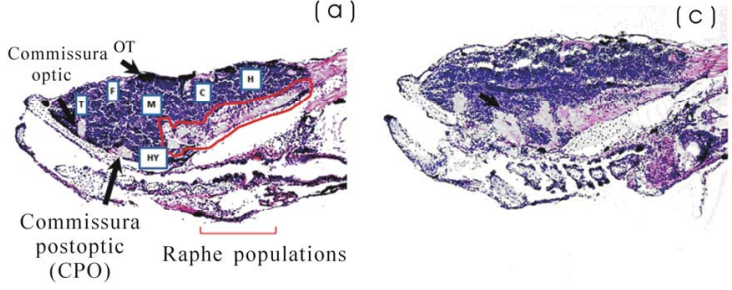

(b)
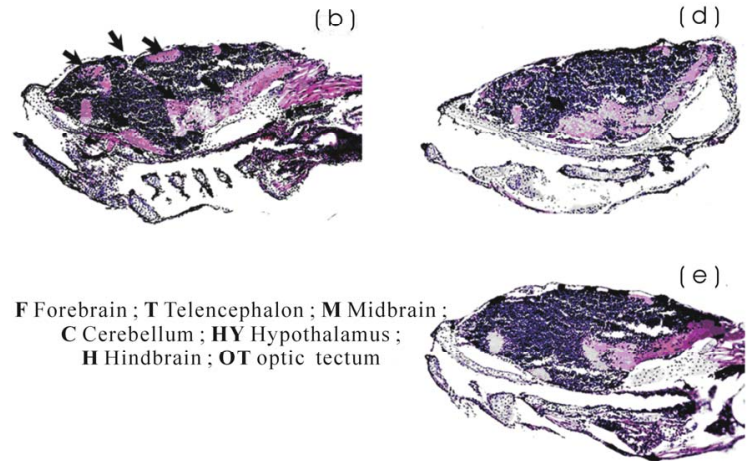

Figure 3. Images of histological sections of brain tissue stained with hematoxylin-eosin. (a) Control; (b) Fluoxetine at 4 dpf; (c) Risperidone at $6 \mathrm{dpf}$; (d) Risperidone at $4 \mathrm{dpf}$; and (e) Fluoxetine at $4 \mathrm{dpf}$ and risperidone at $6 \mathrm{dpf}$. Larvae were analyzed three times $(\mathrm{n}=\mathbf{3})$ at $\mathbf{1 0} \mathrm{dpf}$. In Figure 3(b), the arrows indicate an observable decrease in the number of nucleated areas of the dorsalis telencephali area and the optic tectum; also a larger area in the postoptic commissure and the raphe population zone and a cellular disorganization in the latter. On the other hand, the fissure that separates the midbrain at forebrain increased in size.
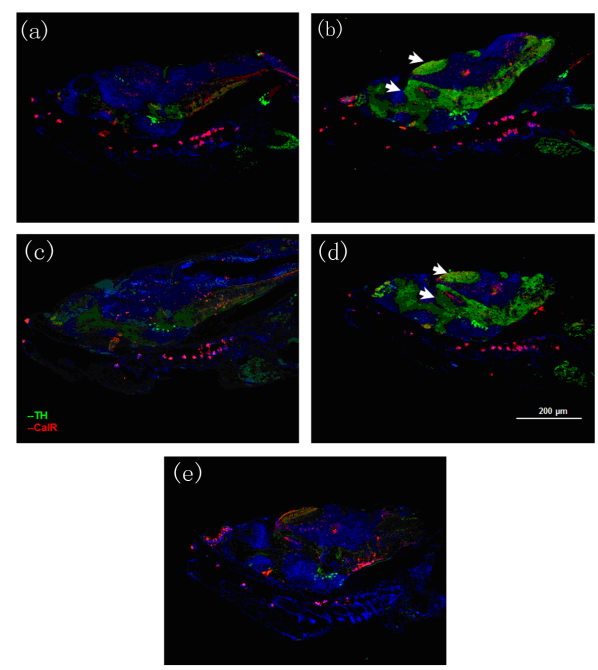

Figure 4. Immunohistochemistry images of brain tissue. Tyrosine hydroxylase, labeled with $\mathrm{Cy} 2$ (green) and Calretinin labeled with $\mathrm{Cy} 3$ (red). (a) Control; (b) Fluoxetine at $4 \mathrm{dpf}$; (c) Risperidone at $6 \mathrm{dpf}$; (d) Risperidone at $4 \mathrm{dpf}$; and (e) Fluoxetine at $4 \mathrm{dpf}$ and risperidone $6 \mathrm{dpf}$. Larvae were analyzed three times $(n=3)$ at $10 \mathrm{dpf}$. The arrows in Figures 4(c) and (d) indicate an increase in the levels of TH + dopaminergic neurons in the dorsal region of the optic tectum of the brain and an increase in the levels of $\mathrm{TH}+$ cells in the midbrain region. 

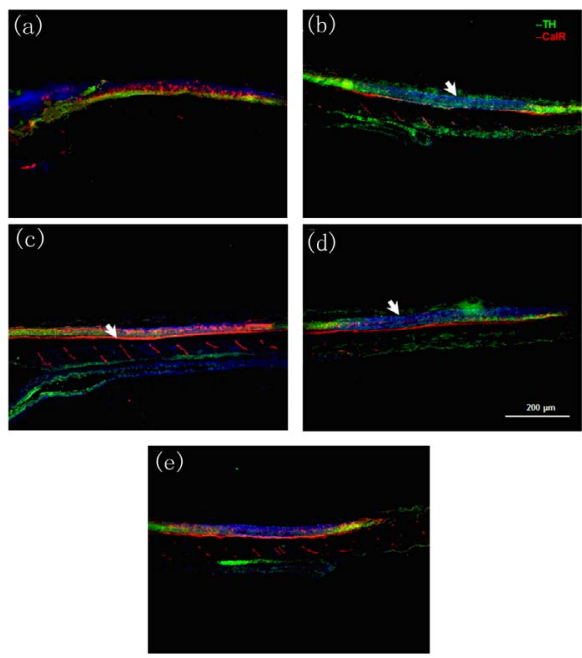

Figure 5. Immunohistochemistry images of spinal cord tissue. Tyrosine hydroxylase, labeled with $\mathrm{Cy2}$ (green) and Calretinin labeled with $\mathrm{Cy3}$ (red). (a) Control; (b) Fluoxetine at $4 \mathrm{dpf}$; (c) Risperidone at $6 \mathrm{dpf}$; (d) Risperidone at 4 dpf; and (e) Fluoxetine at $4 \mathrm{dpf}$ and risperidone at $6 \mathrm{dpf}$. Larvae were analyzed three times $(n=3)$ at 10 dpf. In Figures 5(c) and (d), the arrows indicate a decrease in the levels of CalR-positive motoneurons. In Figures 5(b) and (e) the arrows indicate a change in the width of the nerve fiber formed by the axons of motoneurons.

\section{Discussion}

In this work, the effects of risperidone and fluoxetine on the locomotor activity, heart rate, and brain development of zebrafish larvae were studied. These parameters provide an idea of the effects caused by serotonin in early development stages and provide knowledge on the area of neuropharmacology.

The advantage of studying motoneuron diseases in zebrafish [25] is the rapid development of their spinal cord, which allows the analysis of motoneuron branching patterns as early as $24 \mathrm{hpf}$. In addition, responses to touching and swimming can be monitored after hatching around $48 \mathrm{hpf}$ [26]. It is now recognized that zebrafish show great similarity to mammals and are an extremely useful model for screening compounds at several stages of the drug discovery process $[27,28]$.

The effect of fluoxetine and risperidone exposure was evaluated by examining the heart rate. The results demonstrated that $5 \mu \mathrm{M}$ fluoxetine and/or risperidone-treated larvae exhibit a normal heart rate at 8 and $9 \mathrm{dpf}$ compared to controls. When larvae were exposed to $5 \mu \mathrm{M}$ fluoxetine and/or risperidone at 4 and $6 \mathrm{dpf}$ and for $24 \mathrm{~h}$, SSA was monitored daily from 6 to $9 \mathrm{dpf}$. SSA was significantly lower than controls on the first days after treatments. After $7 \mathrm{dpf}$, SSA levels recovered up to those of control, and were maintained throughout the remaining experimental period under risperidone treatment. In larvae exposed to $5 \mu \mathrm{M}$ fluoxetine alone, SSA was sig- nificantly increased at $9 \mathrm{dpf}$ as compared to controls. However, erratic and abnormal movements were observed with this increase, suggesting a long-term toxic effect of fluoxetine. The unusual body position exhibited in swimming during the behavioral test after exposure to fluoxetine was also observed. While control animals maintained a parallel position with respect to the water surface when swimming, the animals treated with fluoxetine were unable to maintain such a position, indicating lack of postural balance, as described in previous studies [29]. In addition, we found that fluoxetine-treated zebrafish exhibited erratic swimming patterns, manifested by bouts of vertical swimming or sideway swimming, suggesting a coordination problem. This extended effect suggests that fluoxetine effect is not pharmacological, but rather developmental. However, when fish were treated with fluoxetine and risperidone, SSA showed no significant differences with control, and no erratic swimming was observed. This result suggests a reversible process, given that fluoxetine increases the levels of serotonin and risperidone levels out this effect. So, it is important to note that changes in SSA are not pharmacological because for extended periods of time, the effects are reversed when the concentrations of the neurotransmitter are decreased.

Representatives from all major fish classes possess serotonergic neurons in the raphe nuclei, giving rise to ascending and descending pathways [18,30-47]. Due to their apparent resemblance with the mammalian raphe serotonergic neurons, these are the best studied populations within fish. Generally, the assumption for teleosts has been that the two raphe nuclei (superior and inferior raphe) innervate most brain areas in a manner similar to that described for mammals and, accordingly, that they may play equivalent functional roles. Indeed, 5-HT immunoreactive fibers have been detected throughout the fish CNS [30,33,37,39,41,42,48-51].

The raphe serotonergic innervation of the zebrafish dorsal telencephalon, in particular of the lateral zone, is very dense. Further, Lillesaar et al. [18] observed few serotonergic fibers in the medial zone of the dorsal telencephalon originating from the raphe fibers. Interestingly, developmental studies in zebrafish as well as axonal tracing and lesion experiments combined with behavioral assays performed in goldfish suggest that these areas are functionally equivalent to the mammalian hippocampus and amygdala, respectively [52-54]. In mammals, the hippocampus is involved in spatial, contextual, or relational memory and it is known to be supplied by a rich serotonergic innervation mainly from the median raphe populations $[55,56]$.

Several antipsychotic drugs cause a neurotoxic mechanism resulting from an increased or decreased concentration of serotonin both in the synaptic and extracellular 
spaces. In this sense, drug exposure at 4 or $5 \mathrm{dpf}$ coincides with the initial appearance of inferior raphe axons distributed throughout the entire length of the spinal cord in zebrafish [7]. Growth cones of these axons at $4 \mathrm{dpf}$ have been observed adjacent to reticulospinal neurons in the hindbrain and secondary motoneurons in the spinal cord. The temporal correlation between the growth of inferior raphe axons and growth cones throughout the spinal cord and the earliest morphological effects of antipsychotics drugs suggest that raphe axons are affected by the exposure to these drugs. The mechanism of excess or default of serotonin toxicity has been elusive. Considering the temporal correlation between the development of the inferior raphe pathway and risperidone exposure, it was surprising to find no change in SSA. The transient depression of SSA in larvae exposed to risperidone at 4 and $6 \mathrm{dpf}$ may be due to the long half-life of the drug [57]. When the larvae were exposed to fluoxetine alone, first a decrease in swimming and then an increase with erratic movements were noted. However, in zebrafish exposed to fluoxetine at $4 \mathrm{dpf}$ and then to risperidone at 6 dpf, there was no significant change with respect to control, indicating a possible reversal of the effects caused by exposure to fluoxetine observed previously.

In immunohistochemical assays, we used two proteins to determine dopaminergic neurons, labeled with tyrosine hydroxylase (TH) and motoneurons, labeled with calretinin (CalR). TH is the first enzyme in the catecholamine synthesis pathway [58]. TH has also been described to be involved in the stabilization of dopamine synapses [59]. When the larvae were exposed to free risperidone, several changes in the brain were observed. This could be because the drug is a strong blocker of dopamine receptors, which would affect dopaminergic neurons. However, despite the changes observed in the distribution pattern of dopaminergic neurons, these would not affect the SSA. On the other hand, when administered fluoxetine alone or fluoxetine and risperidone, there were no changes with respect to the control. These results indicate that risperidone alone increased the levels of $\mathrm{TH}$ enzymes of the dopaminergic cells when administered as a single drug.

CalR was found in several neuronal populations of the central and peripheral nervous system, primarily in motoneurons and other sensory pathways [60]. CalR is a cytosolic protein of $29-\mathrm{kD}$, belonging to the family of calcium-binding proteins "EF hand" [61], these expressed during early development of the CNS of vertebrates [62], although CalR needs some degree of cell differentiation and tissue to be expressed [63]. Calcium-binding proteins buffer intracellular calcium, thus contributing to the properties of neuronal membranes and their electrical activity. In the spinal cord, we found a decrease in the number of CalR-positive motoneurons in all treatments with free risperidone. These changes could be due to the effect of risperidone on the core neural migration raphe (located in the hypothalamus) to the spinal cord. This migration begins to be observed between 2 and $3 \mathrm{dpf}$ and extends into the caudal spinal cord after 4 days [7]. In the case of fluoxetine, an increase in the width of nerve fiber formed by the axons of motoneurons was observed. This could cause a significant increase in SSA at 9 dpf with erratic and abnormal movements, given the potential neurotoxicity of the drug. In this respect, antipsychotic drugs could alter the extracellular levels of neurotransmitters and thereby modify the development of the CNS [7,14,28,64]. These changes suggest that the neuroanatomy is affected by fluoxetine and risperidone exposure, since these changes are not sufficient to modify the SSA in the case of risperidone.

According to Seibt et al. [28], antipsychotic drugs show high affinity for biomembranes due to their amphiphilic property. This implies that antipsychotic drugs can also interact with membrane lipid organization. The intercalation of antipsychotic drug molecules into the plasma membrane can modify the membrane lipid dynamics, inducing a subsequent modification of the receptor response [64]. Drug interaction elicits short and long range influence on the bilayer structure, conesquently, modulating processes that range from membrane-bound enzyme activity and receptor binding to membrane permeability and transport [65]. The intercalation of antipsychotic drug molecules into the plasma membrane may thus modulate the efficacy and tolerability profile of compounds able to exert their therapeutic effect through their binding with synapse receptors. This suggests that the pharmacological activity of antipsychotic medications may result from a combination of drug-receptor and drug-membrane interactions [66]. Giacomini et al. [29] have suggested that the pharmacological actions of these antipsychotic drugs may be well retained in vertebrates.

Studies in zebrafish will provide an important insight into the side effects of these drugs as well as into the brain control of locomotor activity.

Testing several drug-induced changes in behavioral and serotonin levels is one of the experimental approaches for screening a new therapeutically relevant compounds, and merits further research in this animal model.

\section{Acknowledgements}

This research was supported by a grant from Universidad Nacional de Quilmes. Silvia del Valle Alonso is a member of Consejo Nacional de Investigaciones Científicas y Técnicas (CONICET, Argentina) and IMBICE, CICPBA (Provincia de Buenos Aires, Argentina). M. J. Prieto acknowledges fellows from CONICET, Argentina.

We would also like to thank Instituto de Neurociencias 
de Castilla y León, Facultad de Medicina, Universidad de Salamanca, España, as well as Universidad Nacional de Quilmes and Ministerio Nacional de Ciencia, Tecnología e Innovación Productiva (MINCYT) grants.

\section{REFERENCES}

[1] E. C. Azmitia, "Modern Views on an Ancient Chemical: Serotonin Effects on Cell Proliferation, Maturation, and Apoptosis," Brain Research Bulletin, Vol. 56, No. 5, 2001, pp. 413-424. doi:10.1016/S0361-9230(01)00614-1

[2] J. Lauder and H. Krebs, "Serotonin as a Differentiation Signal in Early Neurogenesis," Developmental Neuroscience, Vol. 1, No. 1, 1978, pp. 15-30. doi:10.1159/000112549

[3] M. J. Prieto, et al., "Optimization and in Vitro Toxicity Evaluation of G4 PAMAM Dendrimer-Risperidone Complexes," European Journal of Medicinal Chemistry, Vol. 46, No. 3, 2011, pp. 845-850. doi:10.1016/j.ejmech.2010.12.021

[4] E. H. Cook Jr., et al., "Evidence of Linkage between the Serotonin Transporter and Autistic Disorder," Molecular Psychiatry, Vol. 2, No. 3, 1997, pp. 247-250. doi:10.1038/sj.mp.4000266

[5] E. H. Cook Jr. and B. L. Leventhal, "The Serotonin System in Autism," Current Opinion in Pediatrics, Vol. 8, No. 4, 1996, pp. 348-354. doi:10.1097/00008480-199608000-00008

[6] M. M. Scott and E. S. Deneris, "Making and Breaking Serotonin Neurons and Autism," International Journal of Developmental Neuroscience, Vol. 23, No. 2-3, 2005, pp. 277-285. doi:10.1016/i.ijdevneu.2004.05.012

[7] M. J. Airhart, et al., "Movement Disorders and Neurochemical Changes in Zebrafish Larvae after Bath Exposure to Fluoxetine (PROZAC)," Neurotoxicology and Teratology, Vol. 29, No. 6, 2007, pp. 652-664. doi:10.1016/j.ntt.2007.07.005

[8] C. R. Marshall, et al., "Structural Variation of Chromosomes in Autism Spectrum Disorder," The American Journal of Human Genetics, Vol. 82, No. 2, 2008, pp. 477-488. doi:10.1016/j.ajhg.2007.12.009

[9] R. P. Malone and A. Waheed, "The Role of Antipsychotics in the Management of Behavioural Symptoms in Children and Adolescents with Autism," Drugs, Vol. 69, No. 5, 2009, pp. 535-548. doi:10.2165/00003495-200969050-00003

[10] E. Courchesne, et al., "Mapping Early Brain Development in Autism," Neuron, Vol. 56, No. 2, 2007, pp. 399413. doi:10.1016/j.neuron.2007.10.016

[11] P. A. McBride, et al., "Serotonergic Responsivity in Male Young Adults with Autistic Disorder. Results of a Pilot Study," Archives of General Psychiatry, Vol. 46, No. 3, 1989, pp. 213-221. doi:10.1001/archpsyc.1989.01810030019003

[12] C. J. McDougle, et al., "Effects of Tryptophan Depletion in Drug-Free Adults with Autistic Disorder," Archives of General Psychiatry, Vol. 53, No. 11, 1996, pp. 993-1000. doi:10.1001/archpsyc.1996.01830110029004

[13] R. C. Casper, et al., "Follow-Up of Children of Depressed Mothers Exposed or Not Exposed to Antidepressant Drugs during Pregnancy," Journal of Pediatrics, Vol. 142, No. 4, 2003, pp. 402-408. doi:10.1067/mpd.2003.139

[14] D. T. Wong, F. P. Bymaster and E. A. Engleman, "Prozac (Fluoxetine, Lilly 110140), the First Selective Serotonin Uptake Inhibitor and an Antidepressant Drug: Twenty Years since Its First Publication," Life Sciences, Vol. 57, No. 5, 1995, pp. 411-441. doi:10.1016/0024-3205(95)00209-O

[15] K. L. Grant, "Investigational Drug Tracking: Phases I-III and NDA Submissions-Part I," Hospital Pharmacy, Vol. 29, No. 9, 1994, pp. 830-836, 839-844, 847-852.

[16] Y. A. Su, et al., "Risperidone Attenuates MK-801-Induced Hyperlocomotion in Mice via the Blockade of Serotonin 5-HT 2A/2C Receptors," European Journal of Pharmacology, Vol. 564, No. 1-3, 2007, pp. 123-130. doi:10.1016/j.ejphar.2007.02.031

[17] B. L. Jacobs and C. A. Fornal, "Serotonin and Motor Activity," Current Opinion in Neurobiology, Vol. 7, No. 6, 1997, pp. 820-825. doi:10.1016/S0959-4388(97)80141-9

[18] C. Lillesaar, "The Serotonergic System in Fish," Journal of Chemical Neuroanatomy, Vol. 41, No. 4, 2011, pp. 294308. doi:10.1016/j.jchemneu.2011.05.009

[19] D. L. McLean and J. R. Fetcho, "Relationship of Tyrosine Hydroxylase and Serotonin Immunoreactivity to Sensorimotor Circuitry in Larval Zebrafish," Journal of Comparative Neurology, Vol. 480, No. 1, 2004, pp. 57-71. doi:10.1002/cne.20281

[20] M. A. Akimenko, et al., "Differential Induction of Four msx Homeobox Genes during Fin Development and Regeneration in Zebrafish," Development, Vol. 121, No. 2, 1995, pp. 347-357.

[21] E. Weruaga, et al., "Distribution of the Calcium-Binding Proteins Parvalbumin, Calbindin D-28k and Calretinin in the Retina of Two Teleosts," Journal of Chemical Neuroanatomy, Vol. 19, No. 1, 2000, pp. 1-15. doi:10.1016/S0891-0618(00)00046-6

[22] D. Clemente, et al., "Effects of Axotomy on the Expression of NADPH-Diaphorase in the Visual Pathway of the Tench," Brain Research, Vol. 925, No. 2, 2002, pp. 183194. doi:10.1016/S0006-8993(01)03279-6

[23] F. J. Arenzana, et al., "Teratogenic Effects of Ethanol Exposure on Zebrafish Visual System Development," Neurotoxicology and Teratology, Vol. 28, No. 3, 2006, pp. 342348. doi:10.1016/i.ntt.2006.02.001

[24] F. J. Arenzana, et al., "Tyrosine Hydroxylase Immunoreactivity in the Developing Visual Pathway of the Zebrafish," Anatomy and Embryology, Vol. 211, No. 4, 2006, pp. 323-334. doi:10.1007/s00429-006-0084-2

[25] E. Kabashi, et al., "Zebrafish Models for the Functional Genomics of Neurogenetic Disorders," Biochimica et Biophysica Acta, Vol. 1812, No. 3, 2010, pp. 335-345.

[26] P. Drapeau, et al., "Development of the Locomotor Network in Zebrafish," Progress in Neurobiology, Vol. 68, No. 2, 2002, pp. 85-111. 
doi:10.1016/S0301-0082(02)00075-8

[27] S. Bretaud, S. Lee and S. Guo, "Sensitivity of Zebrafish to Environmental Toxins Implicated in Parkinson's Disease," Neurotoxicology and Teratology, Vol. 26, No. 6, 2004, pp. 857-864. doi:10.1016/j.ntt.2004.06.014

[28] K. J. Seibt, et al., "Antipsychotic Drugs Inhibit Nucleotide Hydrolysis in Zebrafish (Danio rerio) Brain Membranes," Toxicology in Vitro, Vol. 23, No. 1, 2009, pp. 78-82. doi:10.1016/j.tiv.2008.10.003

[29] N. J. Giacomini, et al., "Antipsychotics Produce Locomotor Impairment in Larval Zebrafish," Anatomy and Embryology, Vol. 28, No. 2, 2006, pp. 245-250. doi:10.1016/j.ntt.2006.01.013

[30] F. Adrio, R. Anadon and I. Rodriguez-Moldes, "Distribution of Serotonin (5HT)-Immunoreactive Structures in the Central Nervous System of Two Chondrostean Species (Acipenser baeri and Huso huso)," Journal of Comparative Neurology, Vol. 407, No. 3, 1999, pp. 333-348. doi:10.1002/(SICI)1096-9861(19990510)407:3<333::AIDCNE3>3.0.CO;2-R

[31] M. Antri, et al., "Ontogeny of 5-HT Neurons in the Brainstem of the Lamprey, Petromyzon marinus," Journal of Comparative Neurology, Vol. 495, No. 6, 2006, pp. 788800. doi: $10.1002 / \mathrm{cne} .20910$

[32] A. Barreiro-Iglesias, et al., "Descending Brain-Spinal Cord Projections in a Primitive Vertebrate, the Lamprey: Cerebrospinal Fluid-Contacting and Dopaminergic Neurons," Journal of Comparative Neurology, Vol. 511, No. 6, 2008, pp. 711-723. doi:10.1002/cne.21863

[33] T. F. Batten, et al., "Immunolocalization of Catecholamine Enzymes, Serotonin, Dopamine and L-Dopa in the Brain of Dicentrarchus labrax (Teleostei)," Brain Research Bulletin, Vol. 31, No. 3-4, 1993, pp. 233-252. doi:10.1016/0361-9230(93)90214-V

[34] V. Bolliet and M. A. Ali, "Immunohistochemical Study of the Development of Serotoninergic Neurons in the Brain of the Brook Trout Salvelinus fontinalis," Brain, Behavior and Evolution, Vol. 40, No. 5, 1992, pp. 234249. doi: $10.1159 / 000113915$

[35] I. Carrera, et al., "Development of the Serotoninergic System in the Central Nervous System of a Shark, the Lesser Spotted Dogfish Scyliorhinus canicula," Journal of Comparative Neurology, Vol. 511, No. 6, 2008, pp. 804-831. doi:10.1002/cne.21857

[36] A. Chiba and S. Oka, "Serotonin-Immunoreactive Structures in the Central Nervous System of the Garfish Lepisosteus productus (Semionotiformes, Osteichthyes)," Neuroscience Letters, Vol. 261, No. 1-2, 1999, pp. 73-76. doi:10.1016/S0304-3940(98)01011-8

[37] M. Corio, J. Peute and H. W. Steinbusch, "Distribution of Serotonin- and Dopamine-Immunoreactivity in the Brain of the Teleost Clarias gariepinus," Journal of Chemical Neuroanatomy, Vol. 4, No. 2, 1991, pp. 79-95. doi:10.1016/0891-0618(91)90033-9

[38] S. A. Johnston, L. Maler and B. Tinner, "The Distribution of Serotonin in the Brain of Apteronotus leptorhynchus: An Immunohistochemical Study," Journal of Chemical Neuroanatomy, Vol. 3, No. 6, 1990, pp. 429-465.

[39] I. A. Khan and P. Thomas, "Immunocytochemical Local- ization of Serotonin and Gonadotropin-Releasing Hormone in the Brain and Pituitary Gland of the Atlantic Croaker Micropogonias undulatus," General and Comparative Endocrinology, Vol. 91, No. 2, 1993, pp. 167180. doi:10.1006/gcen.1993.1116

[40] J. Meek and H. W. Joosten, "Distribution of Serotonin in the Brain of the Mormyrid Teleost Gnathonemus petersii," Journal of Comparative Neurology, Vol. 281, No. 2, 1989, pp. 206-224. doi:10.1002/cne.902810205

[41] J. Pierre, et al., "The Serotoninergic System of the Brain of the Lamprey, Lampetra fluviatilis: An Evolutionary Perspective," Journal of Chemical Neuroanatomy, Vol. 5, No. 3, 1992, pp. 195-219. doi:10.1016/0891-0618(92)90046-S

[42] F. J. Rodriguez-Gomez, et al., "Distribution of Serotonin in the Brain of the Senegalese Sole, Solea senegalensis: An Immunohistochemical Study," Journal of Chemical Neuroanatomy,Vol. 18, No. 3, 2000, pp. 103-115. doi:10.1016/S0891-0618(99)00049-6

[43] S. L. Stuesse and W. L. Cruce, "Distribution of Tyrosine Hydroxylase, Serotonin, and Leu-Enkephalin Immunoreactive Cells in the Brainstem of a Shark, Squalus acanthias," Brain, Behavior and Evolution, Vol. 39, No. 2, 1992, pp. 77-92. doi:10.1159/000114106

[44] S. L. Stuesse, W. L. Cruce and R. G. Northcutt, "Distribution of Tyrosine Hydroxylase- and Serotonin-Immunoreactive Cells in the Central Nervous System of the Thornback Guitarfish, Platyrhinoidis triseriata," Journal of Chemical Neuroanatomy, Vol. 3, No. 1, 1990, pp. 4558.

[45] S. L. Stuesse, W. L. Cruce and R. G. Northcutt, "Localization of Serotonin, Tyrosine Hydroxylase, and LeuEnkephalin Immunoreactive Cells in the Brainstem of the Horn Shark, Heterodontus francisci," Journal of Comparative Neurology, Vol. 308, No. 2, 1991, pp. 277-292. doi:10.1002/cne.903080211

[46] S. L. Stuesse, D. C. Stuesse and W. L. Cruce, "Raphe Nuclei in Three Cartilaginous Fishes, Hydrolagus colliei, Heterodontus francisci, and Squalus acanthias," Journal of Comparative Neurology, Vol. 358, No. 3, 1995, pp. 414-427. doi:10.1002/cne.903580308

[47] T. C. Ritchie, et al., "The Distribution of Serotonin in the CNS of an Elasmobranch Fish: Immunocytochemical and Biochemical Studies in the Atlantic stingray, Dasyatis sabina," Journal of Comparative Neurology, Vol. 221, No. 4, 1983, pp. 429-443. doi:10.1002/cne.902210406

[48] P. Ekstrom, "Developmental Changes in the Brain-Stem Serotonergic Nuclei of Teleost Fish and Neural Plasticity," Cellular and Molecular Neurobiology, Vol. 14, No. 4, 1994, pp. 381-393. doi:10.1007/BF02088718

[49] J. Kaslin and P. Panula, "Comparative Anatomy of the Histaminergic and other Aminergic Systems in Zebrafish (Danio rerio)," Journal of Comparative Neurology, Vol. 440, No. 4, 2001, pp. 342-377. doi:10.1002/cne.1390

[50] O. Kah and P. Chambolle, "Serotonin in the Brain of the Goldfish, Carassius auratus. An Immunocytochemical Study," Cell and Tissue Research, Vol. 234, No. 2, 1983, pp. 319-333. doi:10.1007/BF00213771 
[51] J. Meek, "Functional Anatomy of the Tectum Mesencephali of the Goldfish. An Explorative Analysis of the Functional Implications of the Laminar Structural Organization of the Tectum," Brain Research, Vol. 287, No. 3, 1983, pp. 247-297.

[52] R. G. Northcutt, "Connections of the Lateral and Medial Divisions of the Goldfish Telencephalic Pallium," Journal of Comparative Neurology, Vol. 494, No. 6, 2006, pp. 903-943. doi:10.1002/cne.20853

[53] M. Portavella, et al., "The Effects of Telencephalic Pallial Lesions on Spatial, Temporal, and Emotional Learning in Goldfish," Brain Research Bulletin, Vol. 57, No. 3-4, 2002, pp. 397-399. doi:10.1016/S0361-9230(01)00699-2

[54] M. F. Wullimann and T. Mueller, "Teleostean and Mammalian Forebrains Contrasted: Evidence from Genes to Behavior," Journal of Comparative Neurology, Vol. 475, No. 2, 2004, pp. 143-162. doi:10.1002/cne.20183

[55] H. Imai, D. A. Steindler and S. T. Kitai, "The Organization of Divergent Axonal Projections from the Midbrain Raphe Nuclei in the Rat," Journal of Comparative Neurology, Vol. 243, No. 3, 1986, pp. 363-380. doi:10.1002/cne.902430307

[56] L. P. Morin and E. L. Meyer-Bernstein, "The Ascending Serotonergic System in the Hamster: Comparison with Projections of the Dorsal and Median Raphe Nuclei," Neuroscience, Vol. 91, No. 1, 1999, pp. 81-105.

[57] L. Lemberger, et al., "Fluoxetine: Clinical Pharmacology and Physiologic Disposition," Journal of Clinical Psychiatry, Vol. 46, No. 3, 1985, pp. 14-19.

[58] S. Kanda, et al., "Over-Expression of bHLH Genes Facilitate Neural Formation of Mouse Embryonic Stem (ES) Cells in Vitro," International Journal of Developmental Neuroscience, Vol. 22, No. 3, 2004, pp. 149-156. doi:10.1016/j.ijdevneu.2004.01.002

[59] G. E. Spencer, J. Klumperman and N. I. Syed, "Neuro- transmitters and Neurodevelopment. Role of Dopamine in Neurite Outgrowth, Target Selection and Specific Synapse Formation," Perspectives on Developmental Neurobiology, Vol. 5, No. 4, 1998, pp. 451-467.

[60] B. Pasteels, et al., "Calbindin and Calretinin Localization in Retina from Different Species," Visual Neuroscience, Vol. 5, No. 1, 1990, pp. 1-16. doi: $10.1017 /$ S0952523800000031

[61] J. H. Rogers, "Calretinin: A Gene for a Novel CalciumBinding Protein Expressed Principally in Neurons," The Journal of Cell Biology, Vol. 105, No. 3, 1987, pp. 1343 1353. doi:10.1083/jcb.105.3.1343

[62] R. Guglielmone and G. Corvetti, "First Appearance and Distribution of Calretinin-Immunoreactive Neurons in the Early Development of the Chick Central Nervous System," Cell and Tissue Research, Vol. 300, No. 1, 2000, pp. 21-28. doi:10.1007/s004410050044

[63] M. Dolder, et al., "Crystallization of the Human, Mitochondrial Voltage-Dependent Anion-Selective Channel in the Presence of Phospholipids," Journal of Structural Biology, Vol. 127, No. 1, 1999, pp. 64-71. doi:10.1006/jsbi.1999.4141

[64] C. Tessier, et al., "Modification of Membrane Heterogeneity by Antipsychotic Drugs: An X-Ray Diffraction Comparative Study," Journal of Colloid and Interface Science, Vol. 320, No. 2, 2008, pp. 469-475. doi:10.1016/j.jcis.2008.01.034

[65] M. A. Carfagna and B. B. Muhoberac, "Interaction of Tricyclic Drug Analogs with Synaptic Plasma Membranes: Structure-Mechanism Relationships in Inhibition of Neuronal $\mathrm{Na}+/ \mathrm{K}(+)$-ATPase Activity," Molecular Pharmacology, Vol. 44, No. 1, 1993, pp. 129-141.

[66] A. Jutila, et al., "Comparison of the Effects of Clozapine, Chlorpromazine, and Haloperidol on Membrane Lateral Heterogeneity," Chemistry and Physics of Lipids, Vol. 112, No. 2, 2001, pp. 151-163.

\author{
Abbreviations \\ Hpf: Hours Post Fertilization; \\ Dpf: Days Post Fertilization; \\ SSA: Spontaneous Swim Activity; \\ TH: Tyrosine Hydroxylase; \\ CalR: Calretinin; \\ CNS: Central Nervous System; \\ ASD: Autism Spectrum Disorders; \\ SSRI: Selective Serotonin Reuptake Inhibitor; \\ Risp: Risperidone; \\ LOEC: Lowest Observable Effective Concentration; \\ bpm: Beats Per Minute;
}

PB: Phosphate Buffer $0.1 \mathrm{M}$;

PFA: 4\% v/v Paraformaldehyde;

RT: Room Temperature;

MLF: Medial Longitudinal Fascicle. 